\title{
De novo genome assembly of a foxtail millet cultivar Huagu 11 uncovered the genetic difference to the cultivar Yugu1, and the genetic mechanism of imazethapyr tolerance
}

Jie Wang ${ }^{1,2,3+}$, Shiming $\mathrm{Li}^{2,3+}$, Lei Lan ${ }^{2+}$, Mushan Xie ${ }^{1}$, Shu Cheng ${ }^{2}$, Xiaolong Gan ${ }^{4,5}$, Gang Huang ${ }^{2,3}$, Guohua Du², Kang Yu ${ }^{2,3}$, Xuemei $\mathrm{Ni}^{2^{*}}$, Baolong Liu ${ }^{4,5^{*}}$ and Guoxiong Peng ${ }^{1^{*}}$

\begin{abstract}
Background: Setaria italica is the second-most widely planted species of millets in the world and an important model grain crop for the research of C4 photosynthesis and abiotic stress tolerance. Through three genomes assembly and annotation efforts, all genomes were based on next generation sequencing technology, which limited the genome continuity.

Results: Here we report a high-quality whole-genome of new cultivar Huagu11, using single-molecule real-time sequencing and High-throughput chromosome conformation capture ( $\mathrm{Hi}-\mathrm{C})$ mapping technologies. The total assembly size of the Huagu11 genome was $408.37 \mathrm{Mb}$ with a scaffold N50 size of $45.89 \mathrm{Mb}$. Compared with the other three reported millet genomes based on the next generation sequencing technology, the Huagu11 genome had the highest genomic continuity. Intraspecies comparison showed about 94.97 and $94.66 \%$ of the Yugu1 and Huagu 11 genomes, respectively, were able to be aligned as one-to-one blocks with four chromosome inversion. The Huagu1 1 genome contained approximately $19.43 \mathrm{Mb}$ Presence/absence Variation (PAV) with 627 protein-coding transcripts, while Yugu1 genomes had 20.53 Mb PAV sequences encoding 737 proteins. Overall, 969,596 Single-nucleotide polymorphism (SNPs) and 156,282 insertion-deletion (InDels) were identified between these two genomes. The genome comparison between Huagu11 and Yugu1 should reflect the genetic identity and variation between the cultivars of foxtail millet to a certain extent. The Ser-626-Aln substitution in acetohydroxy acid synthase (AHAS) was found to be relative to the imazethapyr tolerance in Huagu11.
\end{abstract}

Conclusions: A new improved high-quality reference genome sequence of Setaria italica was assembled, and intraspecies genome comparison determined the genetic identity and variation between the cultivars of foxtail millet. Based on the genome sequence, it was inferred that the Ser-626-Aln substitution in AHAS was responsible for the

\footnotetext{
*Correspondence: nixuemei@genomics.cn; blliu@nwipb.cas.cn; gxpeng@cqu.edu.cn

${ }^{\dagger}$ Jie Wang, Shiming Li and Lei Lan contributed equally to this work.

${ }^{1}$ Genetic, Engineering Research Center, School of Life Sciences,

Chongqing University, Chongqing 401331, China

2 BGI Institute of Applied Agriculture, BGI-Shenzhen, Shenzhen 518120,

China

${ }^{5}$ Key Laboratory of Crop Molecular Breeding, Xining 810008, Qinghai,

China

Full list of author information is available at the end of the article
}

(C) The Author(s) 2021. Open Access This article is licensed under a Creative Commons Attribution 4.0 International License, which permits use, sharing, adaptation, distribution and reproduction in any medium or format, as long as you give appropriate credit to the original author(s) and the source, provide a link to the Creative Commons licence, and indicate if changes were made. The images or other third party material in this article are included in the article's Creative Commons licence, unless indicated otherwise in a credit line to the material. If material is not included in the article's Creative Commons licence and your intended use is not permitted by statutory regulation or exceeds the permitted use, you will need to obtain permission directly from the copyright holder. To view a copy of this licence, visit http://creativecommons.org/licenses/by/4.0/. The Creative Commons Public Domain Dedication waiver (http://creativeco mmons.org/publicdomain/zero/1.0/) applies to the data made available in this article, unless otherwise stated in a credit line to the data. 
imazethapyr tolerance in Huagu11. The new improved reference genome of Setaria italica will promote the genic and genomic studies of this species and be beneficial for cultivar improvement.

Keywords: Setaria italica, Genome, Comparative genomic analysis, Acetohydroxy acid synthase, Imazethapyr tolerance

\section{Background}

Foxtail millet (Setaria italic) is a climate-resilient cereal crop domesticated in northern China more than 8000 years ago [1] and is mainly cultivated in arid and semi-arid regions. It also serves as a model crop for the study of $\mathrm{C} 4$ photosynthesis, stress tolerance and bioenergy traits, due to its small genome size and short lifecycle [2-6]. The genome assemblies of two foxtail millet strains, 'Yugul' and 'zhang gu', were published in 2012, which have accelerated the research of biology and genetics of this species [7-9]. The 'Yugu1' genome assembly based on Sanger sequencing, has higher contiguity than the short-read genome assembly of 'Zhang gu' (contig N50: $126.3 \mathrm{~kb}$ versus $25.8 \mathrm{~kb}$ ) and is currently used as a reference genome of foxtail millet. Although the 'Yugu1' reference genome is well-annotated and has been used in many studies, its total length of $401 \mathrm{Mb}$ only covers $\sim 80 \%$ of the estimated genome size ( $510 \mathrm{Mb}$,) based on k-mer analysis and is distributed in 6,778 contigs [9]. The missing sequences are mainly long repeats with lengths of 5-10 kb, which are difficult to assemble at that time using Sanger or next generation sequencing strategy. Although the function of repetitive sequences is seldom researched in foxtail millet, they have proved to play important roles in the regulation of gene expression and genome evolution in other cereal crops [9]. The hard-to-sequence gaps in the scaffolds reside in the intergenic regions and may also contribute to the regulation of gene expression. As quantitative genetic analyses have revealed that many causal variations underlying phenotype changes are from regulatory regions, a complete and high-quality genome assembly is crucial to understand of the genetic mechanisms of climate-resilient features, genome evolution and important agronomic traits in foxtail millet.

The herbicide-resistance trait is an important trait for foxtail millet. Foxtail millet had the relatively small grain, which made the weed problem worse. The weed seeds were similar to the foxtail millet, which was hard to clean the weed seed in seed production. Moreover, the small seed produce the small plant in the germination stage. It is easy to be covered by the weed. The production reduction will be very serious without weed controlling [10]. The use of herbicides is a major measure to control weeds effectively in modern agriculture. Through the original millet cultivars did not have the ability to resist the herbicides, several herbicide-resistance genes had been imported into the modern millet cultivars through the hybridization with wild related species, or chemical mutagenesis. The different herbicide-resistance genes had the resistance to the different herbicide, and the different resistance to the same herbicide [11]. It will be very helpful of understanding the molecular mechanism of the herbicide-resistance for agriculture production. In the breeding process, the breeder usually used several parents to obtain one cultivar with the herbicide-resistance, which lead to trace the resource of the herbicide-resistance gene hardly. The genome assembly of the cultivar will provide an easy way to uncover the mechanism of the herbicide-resistance.

Here, we provide the assembly of a high-quality Huagu11 reference genome through three technologies: single-molecule real-time sequencing (SMRT) chromosome conformation capture sequencing $(\mathrm{Hi}-\mathrm{C})$ and next generation sequencing (NGS). Compared with former cultivars, Huagu11 was planted widely in western China, which should present a special ecotype of Foxtail millet. This new reference genome is helpful for further analysis and comparison of genomic diversity of intraspecific genomes in foxtail millet. By intensively aligning the Yugu1 and Huagu11 genomes, we identified 969,596 SNPs, 156,282 small insertions/deletions (indels, length shorter than $100 \mathrm{bp}$ ) and more than $19 \mathrm{Mb}$ of presence/absence-variation (PAV, length longer than $500 \mathrm{bp}$ ) sequences between these two millet genomes. Interestingly, our comparative genomics analysis revealed a wide range of intraspecific gene-order variations: about 7.54\% of genes were non-syntenic between these two genomes. The two cultivars had different phenotype in the grouting days, plant height, glume color, thousand grain weight, and the response to the imazethapyr (Table S1). Considering the amino acid substitution in AHAS could produce the resistance against the herbicide imazethapyr in several plants [12], the sequences of $A H A S$ were aligned to understand its functional diversity in the two cultivars.

\section{Results \\ Genome sequencing and assembly}

Three technologies were combined to sequence and assembly the Huagu 11 genome: SMRT, $\mathrm{Hi}-\mathrm{C}$ and NGS. In total, we generated $64.43 \mathrm{~Gb}(\sim 155 \times)$ SMRT sequences, $63.31 \mathrm{~Gb}$ high quality clean paired HiSeq reads (PE150) and 34.34 Gb $(\sim 83 \times)$ effective Hi-C reads (Table S2). The K-mer analysis estimated that the genome size of Huagu11 was $456 \mathrm{Mb}$ (Figure S1; 
Table S3). We assembled the genome as the pipeline previously reported. The initial assembly was performed using PacBio SMRT data alone and resulted in 379 contigs with a N50 length of $5.39 \mathrm{Mb}$ (Table 1). The contigs were corrected with the NGS reads and then scaffolded using Hi-C data (Table S4; Figure S2). The total assembly size of the Huagu11 genome is $408.37 \mathrm{Mb}$ (Table 1), which is similar to the recently updated Yugul genome (405.73 Mb). The assembled genome of Huagu11 contains nine scaffolds. The scaffolds number was corresponded to the chromosome number, which meant we got 9 pseudochromosomes from Hi-C. The scaffold N50 size is $45.89 \mathrm{Mb}$ (Table 1), which constituted approximately $98 \%$ of the whole genome. Compared with the other three reported millet genomes, the Huagu11 genome has the minimum number of contigs and the longest contig N50 length, which indicates the highest genomic continuity (Table 1; Table S5).

Three methods were used to evaluate the quality and completeness of the assembled Huagu11 genome. First, approximately $98.3 \%$ (1,351 of 1,375$)$ of embryophyta genes were detected in our assembly using Benchmarking Universal Single-Copy Orthologs (BUSCO) analysis, a percentage similar to that for the Yugu1 genome (97.8\%) (Table S6). Second, while the Hiseq short reads was mapped back to the assembly using BurrowsWheeler-Alignment (BWA) tool with default parameters, about $98.30 \%$ of the total reads could be well mapped, which covered over $99.12 \%$ of the assembly (Table S7). We also found that about $97.75 \%$ of the assembly was covered by more than 20 folds of short NGS reads, which ensured high accuracy of assembly at the single nucleotide level (Table S7). Third, Expressed Sequence Tag (EST) sequences from the NCBI was aligned to the Huagu11 genome assembly by the BLAT software [13] with default parameters. For the 19,441 ESTs longer than $500 \mathrm{bp}$, about $98.61 \%$ of the ESTs could be mapped to the assembled genome, and $95.86 \%$ of the ESTs were credited as complete sequences because at least $90 \%$ of the EST nucleotide could be mapped to one continuous scaffold (Table S8). These results indicated the assembled genome of Huagu11 had a high quality.

\section{Genome annotation}

We used the methods of ab initio structure analysis and homology comparison to search the genome sequences to analyze the repetitive sequences. A total of $182 \mathrm{Mb}$ (44.63\% of the assembly genome) were repetitive sequences (Table S9). The most abundant subtypes were Gypsy-like and Copia-like elements, representing 21.55\% and $10.33 \%$ of the assembly genome, respectively. The remaining transposable elements were DNA transposons (11.53\%), long interspersed nuclear elements (LINEs; 2.46\%), short interspersed nuclear elements (SINEs, $0.13 \%)$, and uncharacterized repeats $(0.83 \%)$ (Table S9).

We annotated the protein coding genes in huagu11 genome by combining the results of protein homology prediction, RNA sequence prediction and ab initio prediction. A total of 42,932 protein coding genes were predicted in the Huagu11 genome, among which 36,652 genes were characterized as high confidence and $65.1 \%$ of the genes could be supported by transcriptome data from four different tissues (Table 1). We annotated the genome and found that $82.5 \%$ of the genes had known functions

Table 1 Summary of genome assembly and annotation

\begin{tabular}{|c|c|c|c|c|}
\hline \multicolumn{5}{|l|}{ Assembly } \\
\hline & & N50 (size/number) & N90 (size/number) & Total length \\
\hline \multirow[t]{3}{*}{ Genome assembly } & Contig & $5.4 \mathrm{Mb} / 25$ & $1.4 \mathrm{Mb} / 86$ & $408 \mathrm{Mb}$ \\
\hline & Scaffold & $45.9 \mathrm{Mb} / 5$ & $36.3 \mathrm{Mb} / 9$ & $408 \mathrm{Mb}$ \\
\hline & Chromosomes & 9 chromosomes (from 173 contig) & & $408 \mathrm{Mb}$ \\
\hline \multicolumn{5}{|l|}{ Annotation } \\
\hline \multirow[t]{4}{*}{ Transposable elements } & Type & & Total length & \\
\hline & Total & & 192 Mb (46.9\%) & \\
\hline & Retroelements & & $141 \mathrm{Mb}(34.57 \%)$ & \\
\hline & DNA transposons & & 47.1 Mb (11.5\%) & \\
\hline \multirow[t]{5}{*}{ Noncoding RNAs } & Type & Copies & Total length (kb) & \\
\hline & miRNA & 161 & 21.4 & \\
\hline & tRNA & 976 & 73.3 & \\
\hline & rRNA & 91 & 10.8 & \\
\hline & snRNA & 503 & 59.2 & \\
\hline \multirow[t]{2}{*}{ Protein coding genes } & Total number & Supported by transcriptome data & Function assigned & High confidence \\
\hline & 41,984 & 23,861 & 35,403 & 36,652 \\
\hline
\end{tabular}


(Table S10). About 41,646 (99.19\%) of the Huagu11 predicted genes were found in nine pseudochromosomes. The protein coding genes were mainly located in the chromosome arms and negatively correlated with density of transposable elements (Fig. 1). The predicted noncoding RNA genes included 91 ribosomal RNA genes, 976 tRNA genes, 161 microRNA (miRNA) genes and 503 small nuclear RNA (snRNA) genes (Table S11).

\section{Intraspecies comparison of the genomes of Yugu1 and Huagu 11}

Interspecies comparison with other species had been made in each published foxtail millet genome. Though, the intraspecies comparison was still absent. Considering the quality of the Yugul genome sequence is very close to the Huagu11, it would be a good opportunity to carry out the interspecies comparison. When we aligned the Huagu11 genome to the Yugul genome in the chromosome scale MUMmer software [14], approximately $94.66 \%$ of the Huagu 11 genome sequence $(381,136,172 \mathrm{bp})$ matched with $94.97 \%$ of theYugu1 genome sequence $(381,111,204$ bp) in one-to-one syntenic block patterns (Fig. 2a).This indicated that most regions of the genomes of these two species are stable. We identified 1858 inversions, 462 intra-translocations, and 631 inter-translocations between these two genomes which account for $\sim 17.61 \mathrm{Mb}$ genome regions in total (Table S12). Four chromosome inversion were observed in the one-to-one syntenic blocks (Fig. 2a). The non-syntenic sequences between the two genomes were mostly transposable elements, and the rest were dispersed genes and intraspecies-specific low-copy sequences.

By comparing the Yugu1 and Huagu11 genome, while only DNA fragments larger than 500 bp were counted, we also identified 18,584 Huagul1-specific genomic segments (19.43 Mb in total) and 18,437 Yugu1-specific genomic segments (20.53 Mb in total). Most of the PAV fragments $99.04 \%$ of Huagu11-specific genomic segments and $98.43 \%$ of Yugu1-specific genomic segments) were shorter than $5 \mathrm{~kb}$ (Figure S3). Only 178 PAV sequences longer than $5 \mathrm{~kb}$ were found in Huagu11, and 294 PAV sequences longer than $5 \mathrm{~kb}$ were found in Yugu1. These PAV sequences were nonuniformly distributed

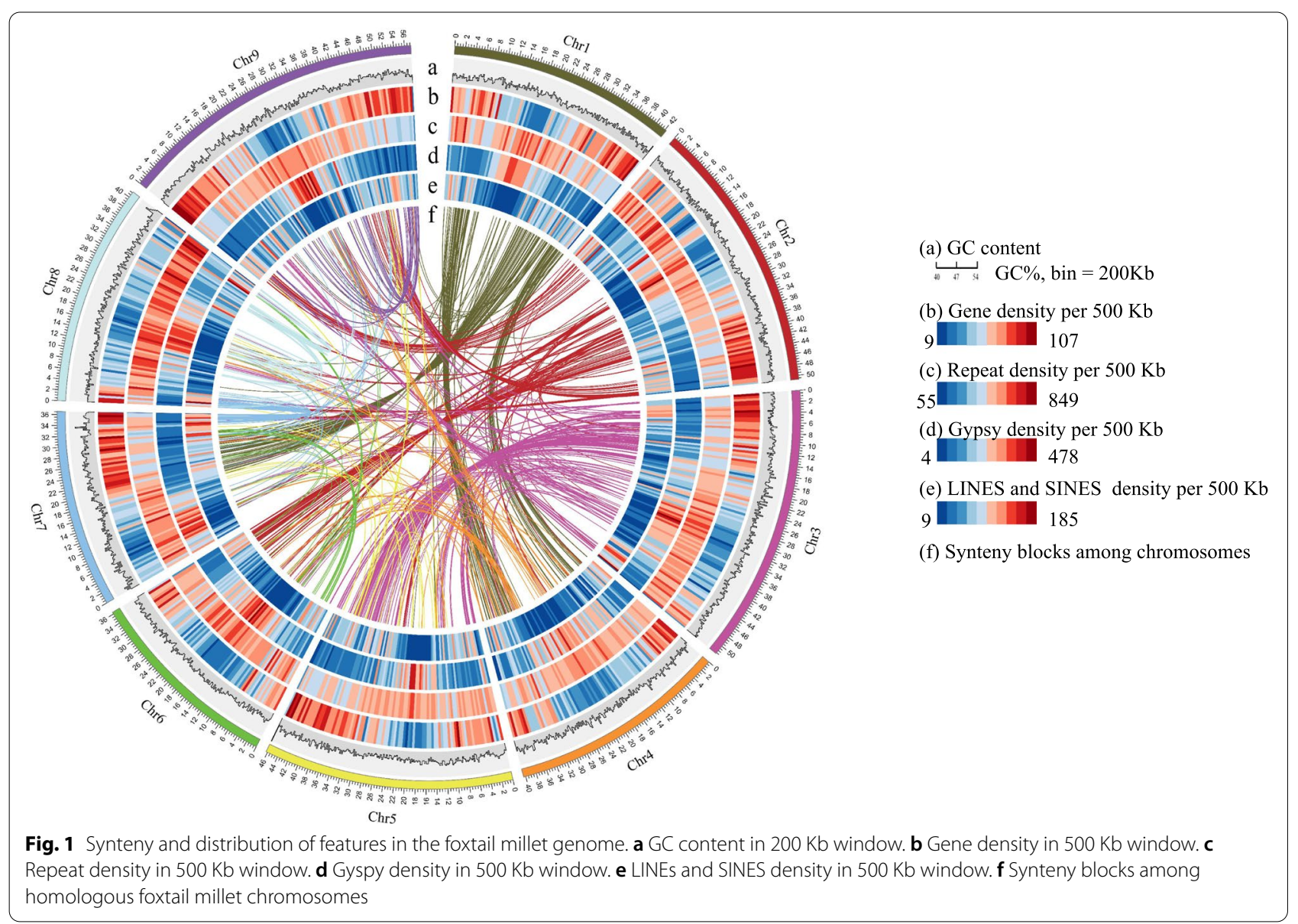




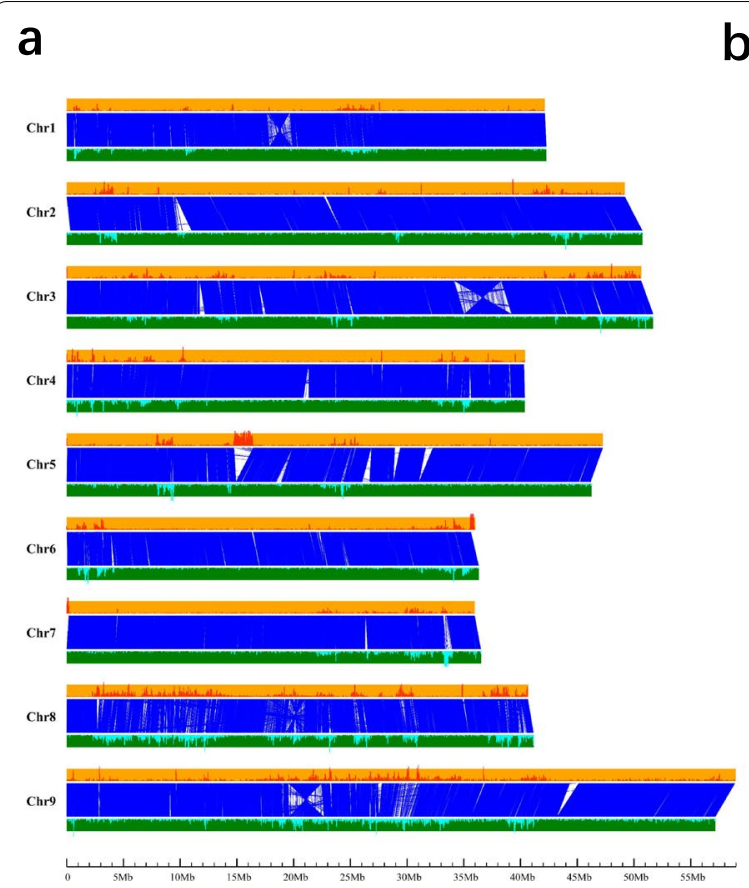

b

Fig. 2 Whole-genome comparison of Yugu1 and Huagu11 genomes. a One-to-one syntenic blocks between Yugu1 and Huagu11 genomes. Orange lines and green lines represent Yugu1 chromosomes and Huagu1 1 chromosomes, respectively. The red plots in the orange lines and the cyan plots in the green lines represent the identified PAV sequences in these two genomes. $\mathbf{b}$ The SNP and indel distributions were aligned the Huagu11 genome to Yugu1 genome by MUMmer software to identify genetic variants. $50 \mathrm{~K}$ bp per window, and cyan lines represent SNP numbers per window and orange lines represent total indels length (bp) per window (including insertions and deletions). The purple lines below the $x$-axis represent SNP-enriched regions greater than $450 \mathrm{~Kb}$. The dark arrow indicated the gene locus of AHAS, which accounted for the herbicide resistant trait of Huagu11

throughout the genome, and some of them were located in clusters (Fig. 2a). Interestingly, there were only $1.12 \mathrm{Mb}$ Yugu1 specific PAV sequences distributed in clusters, while $3.73 \mathrm{Mb}$ Huagu11 specific PAV sequences were distributed in clusters. We identified 627 proteincoding transcripts in Yugu1-specific genomic segments and 737 protein-coding transcripts in Huagu11-specific genomic segments in these PAV regions with the parameters of at least $75 \%$ of coding sequences were overlap with PAV sequences. The annotation and classification of the specific genes showed that the enriched networks of the specific genes of Huagu11 were Plant-pathogen interaction, Indole alkaloid biosynthesis, Betalain biosynthesis, Tyrosine metabolism, Phenylalanine metabolism, Histidine metabolism, Isoquinoline alkaloid biosynthesis, Anthocyanin biosynthesis, Alpha - Linolenic acid metabolism (Figure S4; Table S13). The enriched networks of Yugu1 were Oxidative phosphorylation, Ribosome, Thiamine metabolism, Mismatch repair, Pantothenate and CoA biosynthesis, Base excision repair, Sulfur metabolism, Benzoxazinoid biosynthesis, Endocytosis and Ether lipid metabolism (Figure S5; Table S14). Interestingly, the enriched networks of the specific genes of Huagu11 contained the anthocyanin biosynthesis, which was possibly responsible for the purple glume traits.

OrthoFinder (v2.5.1) was used to identify homologous genes and investigate gene duplication events between Huagu11 and Yugu1 based on an all-verse-all blastp alignment with default parameters $[15,16]$. Four thousand five hundred eighty-three genes were clustered into 917 Huagu11 specific orthologue groups and 1781 genes were clustered into 418 Yug11 specific orthologue groups. One thousand and thirty-seven of single copy gene in Huagu11 were duplicated in Yugu1 with 2,798 duplicated genes (2.70 per gene) and 1,785 of single copy gene in Yugul were duplicated in Huagu11 with 5,750 duplicated genes (3.22 per gene). Eight thousand, six hundred six genes of Huagu11 and 8829 genes of Yugu1 were clustered into 3,031 orthologue groups with more than one gene of both cultivars in the same orthologue group (Table S15).

When we aligned the Huagu11 genome with the Yugu1 pseudochromosomes genome to identify genetic variations between the Yugu1 and Huagu11 genomes, a total of 969,596 SNPs and 156,282 indels (totally 617,674 bp) were identified with an average of 2.42 SNPs and 0.39 
indels $(1.54 \mathrm{bp})$ per kb. There was a positive correlation of the distributions between SNPs and indels (Pearson's correlation $R=0.9859, P<0.001$; Fig. $2 b$ ). We found these SNPs were not evenly distributed on the chromosomes, but clustered together (Figure S6). The windows with SNP density more than 1.5 folds of the average density of the genome ( $\geq 180$ SNPs per $50 \mathrm{~Kb})$ was defined as the SNP enrichment regions. About 1593 windows were identified as SNP-enriched regions which accounted for $19.78 \%$ of the whole genome and contained $89.55 \%$ of SNPs. The windows adjacent in $100 \mathrm{~Kb}$ were merged while the regions more than $450 \mathrm{~kb}$ were illustrated (Fig. 2b). It was a high possibility that these SNP-enriched regions in Huagu11 and Yugu1 were derived from the different genetic resources and contributed to the phenotype diversity in genetics.

\section{The genetic mechanism of the resistance} against the herbicide imazethapyr in Huagu 11

In Yugu1 and Huagu11, there is an obvious difference in the resistance against herbicide imazethapyr (Fig. 3a). The resistance to the herbicide imazethapyr was mainly relative to the AHAS gene in plants [17]. AHAS was found to locate at 24,623,024-24,624,955 bp of chromosome 1 of Huagu11, which was coincidental with the SNP-enriched regions (Fig. 2b). The length of the contig containing the $A H A S$ gene was $6,760,944 \mathrm{bp}$ with $3,606,289$ bp before the translation initiation codon and 3,152,723 bp behind the termination codon, which means the genome sequence of Huagul1 could give the all-round information about AHAS. The sequences of AHAS were picked up from the other three genomes Yugu1, Zhuanggu, and TT8 of Setaria italica [18]. The contig of Yugu 1 was 207,997 bp in length, which was far shorter than Huagu11. The AHAS sequence was incomplete in the Zhanggu genome and was missed in the gene set of the TT8 genome. The phenomenon clearly showed that the quality of the Huagu11 genome was better and more useful than other genomes. Compared with Yugu1, four single nucleotide mutations existed in the AHAS gene of Huagu11 (Figure S6), which caused only one amino acid change in the encoded proteins (Fig. $3 \mathrm{~b}$ ). The amino acid Ser in site 626 of the AHAS protein of Yugu1 was changed into Asn in Huagu11 (Fig. 3b). The
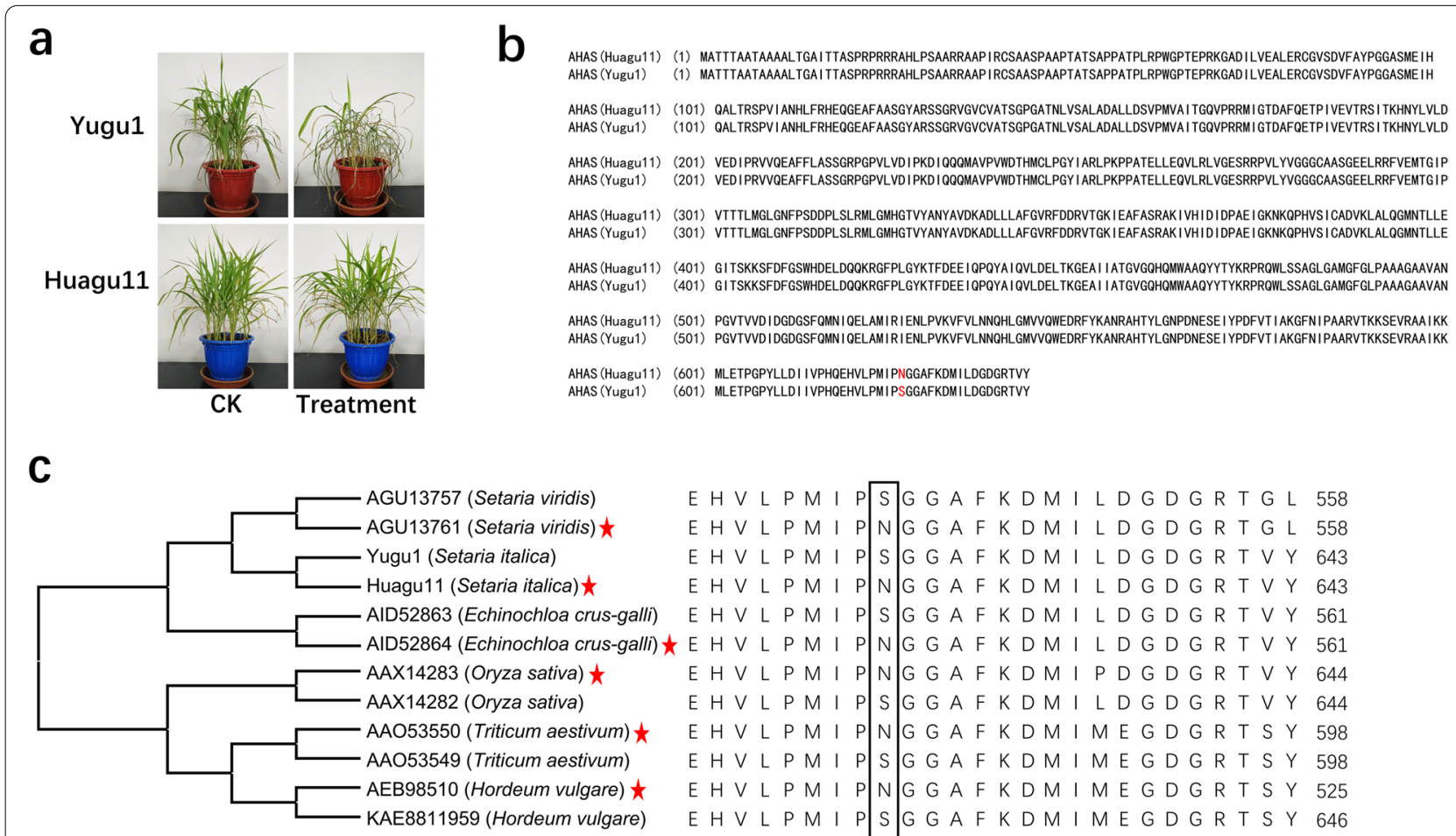

C

$H V L P M I P S G G A F K D M \mid L D G D G R T G L 558$

EHVLPM I P NGGAFKDM I LDGDGRTGL 558

EHVLPMIP S G G A F K DM I LDGDGRTVY 643

EHVLPM I P N G G A F K D M I LDGDGR TVY 643

EHVLPM I P S G G A F K D M I L D GDGRTVY 561

E HVLPM I P N G GA F KDM I LDGDGRTVY 561

EHVLPM I P N G G A F K D M I P D G D G R TVY 644

EHVLPM I P S G GAFKDM I LDGDGRTVY 644

EHVLPM I P NGGAFKDM I MEGDGR T S Y 598

EHVLPM I P S G GAFKDM I MEGDGRT S Y 598

EHVLPMIP NGGAFKDMIMEGDGRTSY 525

E HVLPM I P S G G A F K D M I MEGDGRTS Y 646

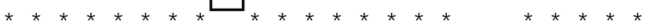

Fig. 3 The phenotype and genetic differences of Huagu1 1 and Yugu1 in the resistance to the herbicide imazethapyr. a The phenotypes of Huagu1 1 and Yugu1 after treatment with the herbicide imazethapyr. The Yugu1 leaves wilted, withered, and died 7 days later after the treatment of $0.25 \%$ Imazethapyr, whereas Huagu 11 grew normally. $\mathbf{b}$ The protein alignment of AHAS from Huagu1 1 and Yugu1. The red letters indicate different amino acids. c Comparative sequence alignment of AHAS showing phylogenetic relationships among members of the grass lineage. The rectangular box indicates the position of the amino acid substitution providing resistance against imidazolinone herbicides. The red * after the cultivars indicates that the cultivar has resistance against imidazolinone herbicides. The * under the sequences indicates the identical position of amino acids in all cultivars 
alleles variation s626n could be found in Setaria viridis, Oryza sativa, Echinochloa crus-galli, Hordeum vulgare, and Triticum aestivum [19-23]. All cultivars carrying Asn had a resistance to imazethapyr in these species, while the cultivars with Ser did not (Fig. 3c). To verify the single nucleotide mutation, the nucleotide sequences of $A H A S$ were amplified and sequenced from the four cultivars and the other two cultivars. The single nucleotide mutation existed exactly in Huagu11 and Y6492 with the herbicide resistance. The remaining cultivars had the same alleles as Yugu1 and were sensitive to the herbicide imazethapyr (Figure S8). The whole coding of AHAS and the SNP of s626n could be found in the transcriptome data of the leaf of Huagu11. All results suggested that s626n was responsible for the resistance to imazethapyr in Huagu11.

\section{Discussion}

The assembly genome of Huagu11 had the highest continuity than previous genomes, which should be attributed to the use of the SMRT sequencing and Hi-C [24]. The genome of Yugu1 was based on the sanger sequencing technology [9], while Zhanggu and TT8 were based on the whole genome shotgun-next-generation sequencing strategy. The SMRT sequencing technology produced high reading length, which made it easy to obtain the longer contigs. The HiC technology could help to produces the longer scaffolds with longer contigs and anchor the longer scaffolds to chromosomes easily. The advanced technologies made the genome assembly economical and convenient. The better reference genome will assist in madding the foxtail millet the model plant for the research of $\mathrm{C} 4$ photosynthesis and abiotic stress tolerance.

The availability of high-quality assembled genomes for both Huagu11 and Yugu1 will provide a distinctive opportunity for in-depth comparison of intraspecific genomes. In one-to-one syntenic blocks, approximately 96\% of the Huagu11 and Yugu1 were mutually syntenic, which was far higher than the value of the B73 and Mo17 maize genomes. Only $60 \%$ of the B73 and Mo17 genomes were able to be aligned as one-to-one blocks [25]. Through the genome sequences of Huagu11 and Yugul had the high syntenic value, extensive PVA, SNP, indel variation, as well as structural variations could be found in the intraspecific comparison. The Yugu1 and Huagu11 genomes each contained approximately $20 \mathrm{Mb}$ of PAV sequence, including 1364 (627 in Yugu1 and 737 in huagu11) high-stringency PAV genes. There were 1858 inversions, 462 intra-translocations, and 631 inter-translocations between these two genomes, which account for $\sim 17.61 \mathrm{Mb}$ genome regions in total. A total of 969,596 SNPs and 156,282 indels (totally $617,674 \mathrm{bp}$ ) were identified between the two cultivars. All these variations could cause the phenotype difference in the two cultivars. For example, a SNP, causing one amino acid change in the $A H A S$ gene, was responsible for the resistance to imazethapyr in Huagu11. Further evaluating the contribution of these differences to the phenotypic variations of agronomic traits would be an interesting future pursuit.

\section{Conclusions}

A new improved high-quality reference genome sequence of Setaria italica was assembled based on SMRT sequencing and $\mathrm{Hi}-\mathrm{C}$ mapping technologies. The total assembly size of the Huagu11 genome was $408.37 \mathrm{Mb}$ with a scaffold N50 size of $45.89 \mathrm{Mb}$, which was the highest genomic continuity of Setaria italica to date. Intraspecies comparison showed about $96 \%$ of the Yugu1 and Huagu11 genomes could be identified as oneto-one blocks with four apparent chromosome inversion. The Huagu11 genome contained approximately 19.43 Mb PAV with 627 protein-coding transcripts, while Yugu1 genomes had 20.53 Mb PAV sequences encoding 737 proteins. There were also 969,596 SNPs and 156,282 indels. Based on the genome sequence of Huagu11, it was found that the Ser-626-Aln substitution in AHAS was responsible for resistance to imazethapyr in Huagu11. The new improved reference genome of Setaria italica will promote the genic and genomic studies of this species and be beneficial for cultivar improvement.

\section{Methods \\ Materials}

The foxtail millet cultivar Huagu11 was bred by the BGI Institute of Applied Agriculture (ShenZhen, China). Huagu11 was derived from the cross of Yugu18 and Y6492 and carried the resistance to the herbicide imazethapyr.

\section{Sampling and sequencing}

The CTAB extraction method was employed for the genomic DNA extraction from the leaf of an individual of the foxtail millet line 'Huagu11' (NCBI Taxonomy ID: 4555) [26]. All sequencing libraries are constructed according to the quality requirements of sequencing equipment. The DNA library was prepared based on the PacBio platform, and the SMRT clock template preparation kit 1.0 (Pacific Biosciences Corporation, California, USA, 100-259-100) was used for the preparation of the PacBio library. The long-read DNA library was sequenced with PacBio RSII sequencer P6-C4 chemical method, and the movie length was $360 \mathrm{~min}$. The Hi-C library was prepared according to the standard procedure [27]. RNA is isolated from different tissues (root, stem and leaf). The 
RNA-seq library was prepared using Illumina platform (Illumina, Santiago, CA, USA). We generated $\sim 84.37 \mathrm{~Gb}$ of paired-end data, the raw genome reads were filtered using SOAPfilter software [28] and $63.31 \mathrm{~Gb}$ of highquality reads were obtained for K-mer analysis and pilon analysis. PacBio sequencing obtained $64.43 \mathrm{~Gb}$ data, with an average read length of 8,657 bp (Table S1). About $132.57 \mathrm{~Gb}$ of raw data was sequenced from a Hi-C library and $\sim 34.34 \mathrm{~Gb}$ of valid reads were obtained after quality control. A total of $10.48 \mathrm{~Gb}, 15.43 \mathrm{~Gb}$ and $10.65 \mathrm{~Gb}$ of RNA-seq raw data were obtained for root, stem and leaf, respectively (Table S1). The raw reads were then filtered using SOAPfilter software, and finally, $10.25 \mathrm{~Gb}$, $14.98 \mathrm{~Gb}, 10.37 \mathrm{~Gb}$ high-quality sequences were obtained for gene prediction (Table S1).

\section{Estimation of the genome size}

$\mathrm{K}$-mer analysis was employed to estimate the genome size. K-mers $(K=17)$ were counted by Jellyfish [29] using 14.5 Gb high-quality short reads. Genome size was estimated according to the formula: Genome Size $=$ K-mer num/Peak depth.

\section{Genome assembly}

After removing the PacBio reads of less than $1 \mathrm{~Kb}$ in length and adapters, $64 \mathrm{~Gb}$ filtered reads, representing $\sim 155 \times$ sequencing coverage of the Huagu11 genome, were used for contig assembly with Falcon v0.3.0 [30]. Then, BLASR [31] was employed to map the readings obtained by PacBio SMRT back to contigs. SMRT was used to correct some sequencing errors. BWA mem was used to map the paired-end reads without Illumina PCR to the corrected contig [32], and further use high-quality reads for assembly [33].

\section{Chromosome assembly using $\mathrm{Hi}-\mathrm{C}$}

The clean $\mathrm{Hi}-\mathrm{C}$ reads were mapped to the genome assembly through BWA align. HiC-Pro was used for repetitive reading removal, classification and quality assessment [34]. The $25.11 \%$ of $\mathrm{Hi}-\mathrm{C}$ data were valid reads. Raw counts of $\mathrm{Hi}-\mathrm{C}$ links were aggregated and separately by using Juicer [35] and 3D-DNA [36]. The Juicebox [37] software was used to adjust the placement and orientation errors where the discrete chromatin interaction patterns were contradictory with the genetic map of Yugu1. According to the threshold of contact frequency, the sequence is divided into 9 groups. A total of 181 scaffolds (representing $98.60 \%$ total length) were anchored to chromosomes in S. italica (Table S3).

\section{Transposable elements annotation}

Both of De novo prediction and homology-based alignment were used to identify transposable elements. The
De novo repeat database was built by a combination of the results of LTR_Finder [38], PILER [39], and RepeatScout [40]. This De novo repeat database together with Repbase [41] were used to identify repeats by RepeatMasker [42] and to identify repeat related proteins by RepeatProteinMask (http://www.repeatmasker.org/). RepeatMasker and TRF [43] were used to annotate the tandem repeats. The results above were combined according to their physical positions and transposable elements were further classified by blast against Repbase.

\section{Gene annotation}

Gene prediction was performed by homology-based prediction, de novo prediction and transcriptome-based prediction. For homology-based prediction, protein sequences from eight species (Brachypodium distachyon, Hordeum vulgare, Oryza sativa, Sorghum bicolor, Setaria viridis, Zea mays, Setaria italica (Zhang gu), and Setaria italica (Yugu1)) (Table S6) were mapped onto the Huagu11 genome by an E-value cutoff of $10^{-5}$, and then Genewise was used for gene structure annotation. AUGUSTUS [44] (Version 2.03) and FGENESH [45] (Version 1.3) were used for de novo prediction. RNA-seq data from three tissues were processed by HISAT2 [46] and StringTie [47] for transcriptomebased prediction. EvidenceModeler [48] software was used to combined the above results and get the final non-redundant reference gene set. InterproScan [49], Gene Ontology (GO) [50], Kyoto Encyclopedia of Genes and Genomes (KEGG), SwissProt [51], TrEMBL and Non-redundant protein NCBI databases were used to annotate the functions of the predicted genes by BLAST searches (E-value cutoff $1 \times 10^{-5}$ ). tRNAscan-SE [52] was used to identify tRNA. Rfam database and Infernal [53] were used to identify noncoding RNAs (including rRNA, miRNA and snRNA) based on homologous alignment.

\section{Technical validation and assessment of the genome assembly and annotation}

Using BWA to map short insert size reads back to assembly, mainly for evaluating the quality of genome assembly. In order to assess the integrity of the genome assembly, EST sequences from the NCBI was mapped to the Huagu11 genome assembly using BLAT with default parameters. To evaluate the completeness of the assembly, BUSCO [54] was executed with default parameters.

\section{Identification of PAV sequences, PAV clusters and PAV genes}

We used an overlapped sliding-windows method to identify the PAV sequences in the Huagu11 and Yugu1 genomes. In order to identify the specific sequences 
of Huagu11, a series of 500 bp short reads with single end were generated by divided the Huagu 11 genome into $500 \mathrm{bp}$ overlapped windows with a step size of $100 \mathrm{bp}$. BWA was used to map these reads to Yugu1 genome with the parameter of "mem - w $500-\mathrm{M}$ ". If the sequences that could not be aligned to Yugu1, or the sequences that could be aligned to Yugul but the coverage was less than $20 \%$, these sequences were merged according to their positions in the Huagu11 genome and were defined as Huagu11-specific sequences. Yugu1-specific sequences were identified in the same way. We compared the length distribution of PAV sequences between Huagu11 and Yugu1 genome and found most of the PAV sequences were less than $2 \mathrm{~Kb}$ (Figure S3). The adjacent PAV sequences (physical distance $<=100 \mathrm{~Kb}$ ) were merged together, and the PAV clusters were defined if more than $10 \%$ of the merged regions were covered by PAV sequences. The genes with more than $75 \%$ of the CDS regions covered by PAV sequences were defined as PAV genes. The function annotation of the PAV genes were obtained by BLAST examine (E-value cutoff $1 \times 10^{-5}$ ) against the InterproScan, Gene Ontology (GO), Kyoto Encyclopedia of Genes and Genomes (KEGG), SwissProt, TrEMBL and Non-redundant protein NCBI databases. Significantly enriched networks were identified using the hypergeometric test [55].

\section{Investigation of homologue genes and duplicated genes between Huagu 11 and Yugu 1}

Protein sequences from Huagu11 and Yugu1 were used to identify homologous genes and investigate gene duplication events by OrthoFinder v2.5.1 with default parameters based on an all-versus-all BLASTP alignment. The genes which could not be assigned into orthologue groups or were clustered into species-specific orthologue groups were considered as speciesspecific. The orthologue groups with one gene from Huagu11 and one gene from Yugu1 were considered as single copy homologous gene pairs. While the orthologue groups with one gene from Huagu11 and more than one gene from Yugu1, we regarded this gene was duplicated in Yugu1 and vice versa. If the gene numbers from one species was more than twice than those from another species, there was a gene duplication event between these two species in this orthologue group.

\section{Identification of chromosomal structural variations}

We used MUMmer v3.23 (http://mummer.sourceforge. net/) to identify the SNPs and InDel between Huagu11 and Yugu1 using the following procedures: (1) Huagu11 genome was used as query genome to align with the reference genome Yugu1 by the nucmer utility under the parameters -mum. (2) the delta-filter utility was used to filter mapping noise and determine the one-to-one alignment blocks with parameters $-1-\mathrm{r}-\mathrm{q}$, SNPs were reported by the show-snps utility under the parameters $-\mathrm{C}-\mathrm{q}-\mathrm{T}$, the adjacent single-base InDels were merged as one InDel.

The detection of inversions and translocations was completed by filtering the nucmer outputs using deltafilter utility through two sets of parameters settings: "-i $90-1-r-q$ " and "- $i 90-g-r-q$ ", respectively, where -1 was used to obtain one-to-one alignment blocks allowing for rearrangements and -g was used to obtain co-linear region which is the global alignment but not allowing rearrangements. These genomic rearrangement regions were finally defined as inversions or translocations depending on their locations and orientations adjacent to their neighboring blocks.

\section{The isolation of AHAS from foxtail millet cultivars}

AHAS was identified from the foxtail millet genomes through BLAST searches (E-value cutoff $1 \times 10^{-5}$ ). The amino acid sequences were aligned with clustalx software [56]. The primers AHAS-F (ATGGCCACGACG ACCGCCGC) and AHAS-R (TCAATACACGGTCCT GCCAT) were designed to amplificated the whole coding sequences of $A H A S$, and the amplification products were 1932 length. Primers were synthesized by BGI Biological Technology Co., Ltd. The $50 \mu$ reaction system included $10 \mu \mathrm{l} 5 \times \mathrm{GC}$ Buffer, $4 \mu \mathrm{l} 10 \mathrm{mmol}$ dNTP, $0.5 \mu \mathrm{l}$ 20 pmol primers, and $0.5 \mu \mathrm{l}(100 \mathrm{ng}$ ) cDNAs (Thermo Fisher Science, Beijing, China) were supplemented with ddH2O. Using high-fidelity Phushion DNA polymerase (Thermo-Fisher Scientific) in the GeneAmp PCR System 9700 (Thermo-Fisher Scientific). The experimental conditions were as follows: one cycle at $98{ }^{\circ} \mathrm{C}$ for $2 \mathrm{~min}, 35$ cycles at $98^{\circ} \mathrm{C}$ for $10 \mathrm{~s}, 65^{\circ} \mathrm{C}$ for $30 \mathrm{~s}$ and $72{ }^{\circ} \mathrm{C}$ for $2 \mathrm{~min}$, followed by a cycle at $72{ }^{\circ} \mathrm{C}$ for $7 \mathrm{~min}$. The PCR products were extracted from $1.0 \%$ agarose gels and cloned into the pGEM-T Easy Vector plasmid. The recombinant plasmid was then transformed into Escherichia coli DH5 $\alpha$ cells and positive clones were sent to Shanghai Shenggong Biological Co., Ltd. for sequencing.

\footnotetext{
Abbreviations

Hi-C: High-throughput chromosome conformation capture; PAV: Presence/ absence Variation; SNP: Single-nucleotide polymorphism; InDels: Insertiondeletion; AHAS: Acetohydroxy acid synthase; SMRT: Single-molecule real-time sequencing; BUSCO: Benchmarking Universal Single-Copy Orthologs; BWA: Burrows-Wheeler-Alignment; EST: Expressed Sequence Tag; LINEs: Long interspersed nuclear elements; SINEs: Short interspersed nuclear elements; miRNA:
} 
MicroRNA; snRNA: Small nuclear RNA; PCR: Polymerase chain reaction; GO: Gene Ontology; KEGG: Kyoto Encyclopedia of Genes and Genomes.

\section{Supplementary Information}

The online version contains supplementary material available at https://doi. org/10.1186/s12870-021-03003-8.

Additional file 1: Figure S1. K-mer analysis to estimate the genome size of Huagu11.

Additional file 2: Figure S2. Hi C linkage density heat map of assembled contigs.

Additional file 3: Figure S3. Length distribution of PAV sequences between Huagu1 1 and Yugu1 genome. Most of the fragments were less than $5 \mathrm{~Kb}$.

Additional file 4: Figure S4. The enriched networks of the specific genes of Huagu11.

Additional file 5: Figure S5. The enriched networks of the specific genes of Yugu1.

Additional file 6: Figure S6. The proportion of the large chromosome segments with genetics difference in the chromosome. Chr8, Chr9 and $\mathrm{Chr} 3$ had relatively high percentages.

Additional file 7: Figure S7. The nucleotide sequence alignment of AHAS from the genome of Huagu1 1 and Yugu1.

Additional file 8: Figure S8. The amino sequence alignment of AHASs from the cultivars with the different resistance to the imazethapyr.

Additional file 9: Table S1. The main different phenotypes of Yugu1 and Huagu11.

Additional file 10: Table S2. List of sequencing data generated.

Additional file 11: Table S3. The statistics of 17-mer analysis.

Additional file 12: Table S4. The statistics of $\mathrm{Hi}-\mathrm{C}$ result.

Additional file 13: Table S5. Comparison of the assemblied genomes among the Huagu11, Yugu, Zhanggu and TT8.

Additional file 14: Table S6. Genome assembly completeness evaluation with BUSCO groups.

Additional file 15: Table S7. Mapping reads to the genome assembly.

Additional file 16: Table S8. Assessment of the Huagu11 genome assembly using 29399 EST sequences.

Additional file 17: Table S9. Repeat element in the foxtail millet genome

Additional file 18: Table S10. Gene function annotation in the foxtail millet genome.

Additional file 19: Table S11. Non-coding RNA annotation in the foxtail millet genome.

Additional file 20: Table S12. Genomic rearrangement details between Huagu11 and Yugu1 genome.

Additional file 21: Table S13. Functional annotation of Huagu11 PAV spefific genes with $\mathrm{Nr}$ (20170924), KEGG (v89.1), Interpro (interproscan-5.30-69.0) and GO (gene_ontology.1_2) database.

Additional file 22: Table S14. Functional annotation of Yugu1 PAV spefific genes with Nr (20170924), KEGG (v89.1), Interpro (interproscan-5.30-69.0) and GO (gene_ontology.1_2) database.

Additional file 23: Table S15. Statistics of gene numbers in the different orthologue groups.

\section{Acknowledgements}

We are gratefully to the two reviewers and editors assigned by the BMC Plant Biology journal for carefully reviewing our manuscript and providing us with some valuable suggestions. We would like to thank Northwest Institute of Plateau Biology for their supports.

\section{Authors' contributions}

$G P, B L$, and XN conceived and designed the experiments. JW, GH, MX, and GD performed the experiments, JW, SL, LL and XG analyzed the data. JW and SL wrote the manuscript, BL and GP reviewed and revised the paper. All authors have read and approved the manuscript.

\section{Funding}

The work was supported by Science, Technology and Innovation Commission of Shenzhen Municipality of China under grant NO.

ZYC201105170390A and special funds for Science, Technology, Innovation and Industrial Development of Shenzhen Dapeng New District (Grant No. KJYF202001-11). The funding agencies played no role in the design of the study and collection, analysis and interpretation of data and in writing the manuscript.

\section{Availability of data and materials}

The data that support the findings of this study have been deposited into CNGB Sequence Archive (CNSA) of China National GeneBank DataBase (CNGBdb) with accession number CNP0000993 (https://db.cngb.org/search/proje ct/CNP0000993/).

\section{Declarations}

Ethics approval and consent to participate

Not applicable.

\section{Consent for publication}

Not applicable.

\section{Competing interests}

The authors and BGI-shenzhen company declare that they have no competing interests.

\section{Author details}

${ }^{1}$ Genetic, Engineering Research Center, School of Life Sciences, Chongqing University, Chongqing 401331, China. ${ }^{2}$ BGI Institute of Applied Agriculture, BGI-Shenzhen, Shenzhen 518120, China. ${ }^{3}$ BGI-Agro Seed Service (Wuhan) Co., Ltd, BGI-Shenzhen, Wuhan 430090, China. ${ }^{4}$ Key Laboratory of Adaptation and Evolution of Plateau Biota, Northwest Institute of Plateau Biology, Chinese Academy of Sciences, Xining 810008, Qinghai, China. ${ }^{5}$ Key Laboratory of Crop Molecular Breeding, Xining 810008, Qinghai, China.

Received: 24 November 2020 Accepted: 4 May 2021

Published online: 12 June 2021

\section{References}

1. Barton L, Newsome SD, Chen F-H, Wang H, Guilderson TP, Bettinger RL. Agricultural origins and the isotopic identity of domestication in northern China. PNAS. 2009;106(14):5523.

2. Brutnell TP, Wang L, Swartwood K, Goldschmidt A, Jackson D, Zhu XG, Kellogg E, Van Eck J. Setaria viridis: a model for C4 photosynthesis. Plant Cell. 2010;22(8):2537-44

3. Doust AN, Kellogg EA, Devos KM, Bennetzen JL. Foxtail millet: a sequence-driven grass model system. Plant Physiol. 2009;149(1):137-41.

4. Li PH, Brutnell TP. Setaria viridis and Setaria italica, model genetic systems for the Panicoid grasses. J Exp Bot. 2011;62(9):3031-7.

5. Muthamilarasan M, Khan Y, Jaishankar J, Shweta S, Lata C, Prasad M. Integrative analysis and expression profiling of secondary cell wall genes in C-4 biofuel model Setaria italica reveals targets for lignocellulose bioengineering. Front Plant Sci. 2015;6:965.

6. Muthamilarasan M, Singh RK, Suresh BV, Rana S, Dulani P, Prasad M. Genomic dissection and expression analysis of stress -responsive genes in C 4 panicoid models, Setaria italica and Setaria viridis. J Biotechnol. 2020;318:57-67.

7. Lata C, Gupta S, Prasad M. Foxtail millet: a model crop for genetic and genomic studies in bioenergy grasses. Crit Rev Biotechnol. 2013;33(3):328-43. 
8. Zhang G, Liu X, Quan Z, Cheng S, Xu X, Pan S, Xie M, Zeng P, Yue Z, Wang $W$, et al. Genome sequence of foxtail millet (Setaria italica) provides insights into grass evolution and biofuel potential. Nat Biotechnol. 2012;30:549.

9. Bennetzen JL, Schmutz J, Wang H, Percifield R, Hawkins J, Pontaroli AC, Estep M, Feng L, Vaughn JN, Grimwood J, et al. Reference genome sequence of the model plant Setaria. Nat Biotechnol. 2012;30(6):555-61.

10. Li Z, Jing X, Li H, Gang T, Xin L, Mu T. Safety and weed control efficiency of Foxtail Millet seedling stage herbicides. Crops. 2017;1 (5):155-9.

11. Zhijiang. Discovery and application of herbicide resistant gene in Foxtail Millet. Genomics Appl Biol. 2010;294:768-74.

12. Xia W, Pan L, Li J, Wang Q, Feng Y, Dong L. Molecular basis of ALS- and/ or ACCase-inhibitor resistance in shortawn foxtail (Alopecurus aequalis Sobol.). Pestic Biochem Physiol. 2015;122:76-80.

13. Birney E, Clamp M, Durbin R. GeneWise and genomewise. Genome Res. 2004;14(5):988-95.

14. Kanehisa M, Goto S. KEGG: kyoto encyclopedia of genes and genomes. Nucleic Acids Res. 2000;28(1):27-30.

15. Emms DM, Kelly S. OrthoFinder: phylogenetic orthology inference for comparative genomics. Genome Biol. 2019;20(1):238.

16. Emms DM, Kelly S. OrthoFinder: solving fundamental biases in whole genome comparisons dramatically improves orthogroup inference accuracy. Genome Biol. 2015;16(157):157.

17. Yu Q, Powles SB. Resistance to AHAS inhibitor herbicides: current understanding. Pest Manag Sci. 2014;70(9):1340-50.

18. Tsai KJ, Lu MYJ, Yang KJ, Li MY, Teng YC, Chen S, Ku MSB, Li WH. Assembling the Setaria italica L. Beauv. genome into nine chromosomes and insights into regions affecting growth and drought tolerance. Sci Rep-Uk. 2016;6(1):35076.

19. Laplante J, Rajcan I, Tardif FJ. Multiple allelic forms of acetohydroxyacid synthase are responsible for herbicide resistance in Setaria viridis. Theor Appl Genet. 2009;119(4):577-85.

20. Rajguru SN, Burgos NR, Shivrain VK, Stewart JM. Mutations in the red rice ALS gene associated with resistance to imazethapyr. Weed Sci. 2005;53(5):567-77.

21. Matzenbacher FO, Bortoly ED, Kalsing A, Merotto AJ. Distribution and analysis of the mechanisms of resistance of barnyardgrass (Echinochloa crus-galli) to imidazolinone and quinclorac herbicides. J Agric Sci. 2015;153(6):1-15.

22. Pozniak CJ, Hucl PJ. Genetic analysis of imidazolinone resistance in mutation-derived lines of common wheat. Crop Sci. 2004;44(1):23-30.

23. Lee H, Rustgi S, Kumar N, Burke I, Yenish JP. Single nucleotide mutation in the barley acetohydroxy acid synthase (AHAS) gene confers resistance to imidazolinone herbicides. PNAS. 2011:108(21):8909-13.

24. Ma J, Xiang Y, Xiong Y, Lin Z, Xue Y, Mao M, Sun L, Zhou Y, Li XZ, Huang. SMRT sequencing analysis reveals the full-length transcripts and alternative splicing patterns in Ananas comosus var. bracteatus. Peer J. 2019;7:e7062.

25. Sun SL, Zhou YS, Chen J, Shi JP, Zhao HM, Zhao HN, Song WB, Zhang M, Cui Y, Dong XM, et al. Extensive intraspecific gene order and gene structural variations between Mo17 and other maize genomes. Nat Genet. 2018;50(9):1289-95.

26. Murray MG, Thompson WF. Rapid isolation of high molecular weight plant DNA. Nucleic Acids Res. 1980;8(19):4321-6.

27. Lieberman-Aiden E, Van Berkum NL, Williams L, Imakaev M, Ragoczy T, Telling A, Amit I, Lajoie BR, Sabo PJ, Dorschner MO. Comprehensive mapping of long-range interactions reveals folding principles of the human genome. Science. 2009:326(5950):289-93.

28. Luo R, Liu B, Xie Y, Li Z, Huang W, Yuan J, He G, Chen Y, Pan Q, Liu Y, et al. SOAPdenovo2: an empirically improved memory-efficient short-read de novo assembler. Gigascience. 2012;1 (1):18.

29. Marçais G, Kingsford C. A fast, lock-free approach for efficient parallel counting of occurrences of k-mers. Bioinformatics. 2011;27(6):764-70.

30. Chin C-S, Peluso P, Sedlazeck FJ, Nattestad M, Concepcion GT, Clum A, Dunn C, O'Malley R, Figueroa-Balderas R, Morales-Cruz A, et al. Phased diploid genome assembly with single-molecule real-time sequencing. Nat Methods. 2016;13:1050

31. Chaisson MJ, Tesler G. Mapping single molecule sequencing reads using basic local alignment with successive refinement (BLASR): application and theory. BMC Bioinformatics. 2012;13(1):238.

32. Li H. Aligning sequence reads, clone sequences and assembly contigs with BWA-MEM. ArXiv 2013;1303.

33. Walker BJ, Abeel T, Shea T, Priest M, Abouelliel A, Sakthikumar S, Cuomo CA, Zeng Q, Wortman J, Young SK, et al. Pilon: an integrated tool for comprehensive microbial variant detection and genome assembly improvement. PLoS ONE. 2014;9(11):e112963.

34. Servant N, Varoquaux N, Lajoie BR, Viara E, Chen C-J, Vert J-P, Heard E, Dekker J, Barillot E. HiC-Pro: an optimized and flexible pipeline for Hi-C data processing. Genome Biol. 2015;16(1):259.

35. Durand NC, Shamim MS, Machol I, Rao SSP, Huntley MH, Lander ES, Aiden EL. Juicer provides a one-click system for analyzing loop-resolution $\mathrm{Hi}-\mathrm{C}$ experiments. Cell Syst. 2016;3(1):95-8.

36. Dudchenko O, Batra SS, Omer AD, Nyquist SK, Hoeger M, Durand NC, Shamim MS, Machol I, Lander ES, Aiden AP, et al. De novo assembly of the Aedes aegypti genome using $\mathrm{Hi}-\mathrm{C}$ yields chromosome-length scaffolds. Science. 2017;356(6333):92

37. Durand NC, Robinson JT, Shamim MS, Machol I, Mesirov JP, Lander ES, Aiden EL. Juicebox provides a visualization system for $\mathrm{Hi}-\mathrm{C}$ contact maps with unlimited zoom. Cell Syst. 2016;3(1):99-101.

38. Xu Z, Wang H. LTR_FINDER: an efficient tool for the prediction of full-length LTR retrotransposons. Nucleic Acids Res. 2007;35(suppl_2):W265-8.

39. Edgar RC, Myers EW. PILER: identification and classification of genomic repeats. Bioinformatics. 2005;21(suppl 1):i152-8.

40. Price AL, Jones NC, Pevzner PA. De novo identification of repeat families in large genomes. Bioinformatics. 2005;21_suppl_1):i351-8.

41. Tarailo-Graovac M, Chen N. Using RepeatMasker to identify repetitive elements in genomic sequences. Curr Protoc Bioinformatics. 2009;25(1):4.10.11-14.10.14.

42. Jurka J, Kapitonov WV, Pavlicek A, Klonowski P, Kohany O, Walichiewicz J. Repbase update, a database of eukaryotic repetitive elements. Cyto Genome Res. 2005;1 10(1-4):462-7.

43. Benson G. Tandem repeats finder: a program to analyze DNA sequences. Nucleic Acids Res. 1999;27(2):573-80.

44. Stanke M, Steinkamp R, Waack S, Morgenstern B. AUGUSTUS: a web server for gene finding in eukaryotes. Nucleic Acids Res. 2004;32(suppl_2):W309-12.

45. Salamov AA, Solovyev VV. Ab initio gene finding in drosophila genomic DNA. Genome Res. 2000;10(4):516-22.

46. Kim D, Langmead B, Salzberg SL. HISAT: a fast spliced aligner with low memory requirements. Nat Methods. 2015;12:357.

47. Pertea M, Pertea GM, Antonescu CM, Chang T-C, Mendell JT, Salzberg SL. StringTie enables improved reconstruction of a transcriptome from RNAseq reads. Nat Biotechnol. 2015;33:290.

48. Haas BJ, Salzberg SL, Zhu W, Pertea M, Allen JE, Orvis J, White O, Buell CR, Wortman JR. Automated eukaryotic gene structure annotation using EVidenceModeler and the program to assemble spliced alignments. Genome Biol. 2008;9(1):R7.

49. Quevillon E, Silventoinen V, Pillai S, Harte N, Mulder N, Apweiler R, Lopez R. InterProScan: protein domains identifier. Nucleic Acids Res. 2005;33(Web Server issue):W116-20.

50. Ashburner M, Ball CA, Blake JA, Botstein D, Butler H, Cherry JM, Davis AP, Dolinski K, Dwight SS, Eppig JT, et al. Gene Ontology: tool for the unification of biology. Nat Genet. 2000;25(1):25-9.

51. The UniProt C. The universal protein resource (UniProt). Nucleic Acids Res. 2006;35(suppl_1):D193-7.

52. Lowe TM, Eddy SR. tRNAscan-SE: A program for improved detection of transfer RNA genes in genomic sequence. Nucleic Acids Res. 1997;25(5):955-64.

53. Nawrocki EP, Kolbe DL, Eddy SR. Infernal 1.0: inference of RNA alignments. Bioinformatics. 2009;25(10):1335-7.

54. Simão FA, Waterhouse RM, loannidis P, Kriventseva EV, Zdobnov EM. BUSCO: assessing genome assembly and annotation completeness with single-copy orthologs. Bioinformatics. 2015;31(19):3210-2.

55. Rivals I, Personnaz L, Taing L, Potier MC. Enrichment or depletion of a GO category within a class of genes: which test? Bioinformatics. 2007;23(4):401-7.

56. Larkin MA, Blackshields G, Brown NP, Chenna R, McGettigan PA, McWilliam H, Valentin F, Wallace IM, Wilm A, Lopez R, et al. Clustal W and Clustal X version 2.0. Bioinformatics. 2007;23(21):2947-8.

\section{Publisher's Note}

Springer Nature remains neutral with regard to jurisdictional claims in published maps and institutional affiliations. 